\title{
Preservation of Architectural Heritage through 3D digitization
}

\author{
George Pavlidis \\ Research Center ATHENÀ \\ Cultural and Educational Technology Institute \\ 58 Tsimiski Str., Xanthi, 67100, Greece \\ gpavlid@ceti.gr \\ Despoina Tsiafakis \\ Research Center ATHENÀ \\ Cultural and Educational Technology Institute \\ 58 Tsimiski Str., Xanthi, 67100, Greece \\ tsiafaki@ceti.gr \\ Vassilios Tsioukas \\ Democritus University of Thrace \\ Department of Architectural Engineering \\ New Building of Central Library, Kimmeria, Xanthi, 67100, Greece \\ vtsiouka@arch.duth.gr \\ Anestis Koutsoudis \\ Research Center ATHENÀ \\ Cultural and Educational Technology Institute \\ 58 Tsimiski Str., Xanthi, 67100, Greece \\ akoutsou@ceti.gr \\ Fotis Arnaoutoglou \\ Research Center ATHENÀ \\ Cultural and Educational Technology Institute \\ 58 Tsimiski Str., Xanthi, 67100, Greece \\ fotarny@ceti.gr \\ Christodoulos Chamzas \\ Democritus University of Thrace \\ Department of Electrical and Computer Engineering \\ 12 Vas. Sofias Str, Xanthi, 67100, Greece \\ chamzas@ee.duth.gr
}

\begin{abstract}
Continuity and relation to the past are inherent in human nature. The remains of the past constitute culture and cultural heritage. Preservation and dissemination of cultural heritage appear to be, nowadays, a universal priority. Historic/architectural monuments are among the most significant categories of cultural heritage and their $3 \mathrm{D}$ digitization appears to be a chief way towards that direction. 3D digitization of architectural heritage is a very specific problem in the digitization domain. Since size, budget and applicability are some of the most important factors in choosing an appropriate digitization method, and since there is not an all-in-one solution in digitization, this problem cannot be always addressed by using
\end{abstract}


one technique. In this paper we review methods that are available for 3D digitization of architectural heritage and we present two case studies of real-world digitization projects involving monuments and urban areas.

\section{INTRODUCTION}

"Architecture" is the art or craft -depending on the different time periods- of the "architect", a word that derives from the ancient Greek "architekton" meaning the "master builder". To the classical Antiquity, architecture meant much more than construction of buildings. According to the earliest surviving work on the subject, 'De Architectura', written by the Roman architect Vitruvius “...Architecture consists of Order, and of Arrangement, and of Proportion and Symmetry and Propriety and Distribution”. Worth of note is also that he adds that the architect should be well versed in fields such as music and astronomy.

The construction of various types of buildings was one of the earliest crafts appeared in the society in order to cover primary human needs (e.g. shelter, security, worship). Early human settlements were essentially rural. As surplus of production began to occur, rural societies transformed into urban ones and cities began to evolve. Over time the complexity of buildings and their types increased. At the same time architecture was related and reflected different aspects of life and society.

Through monuments, buildings and urban planning is expressed the human history and the different civilizations. Architectural heritage is one of the most important aspects of cultural heritage and of outstanding interest and therefore need to be preserved as part of the world heritage of mankind as a whole. Aside from the traditional methods in preservation and presentation of architectural structures, new technologies can contribute significantly to that direction. Digital recording of monuments, buildings, sites, and cities may also help in the preservation, presentation and dissemination of architectural heritage. The necessity for recording cultural heritage monuments is proven by the existence of international organizations that have, as main target, the definition of strict specifications for the monuments' proper documentation. According to ICOMOS every national and international organization that is responsible for manmade monuments is also responsible to accomplish a proper documentation using appropriate methods: "Such methods might include written descriptions and analyses, photographs (aerial or terrestrial), rectified 
photography, photogrammetry, geophysical survey, maps, measured plans, drawings and sketches, replicas or other traditional and modern technologies"1.

Architectural monuments are part of a global but also a local cultural heritage and their digitization in 3D is a specific problem in the digitization domain. Unfortunately, this problem (as almost all digitization problems) cannot be always addressed by using one digitization technique. The plethora of available 3D digitization techniques is the result of three main properties that influence the suitability and the applicability of a method:

1. Complexity in size and shape

2. Morphological complexity (level of detail)

3. Diversity of raw materials

Additionally, size, budget and applicability are some of the most important factors in choosing an appropriate digitization method, and since there is not an all-in-one solution in digitization, the problem of 3D digitization of architectural heritage cannot be always addressed by using one technique. Various techniques have been proposed and different technologies have been developed: some based on laser scanning, others on surveying-photogrammetric techniques, some using simple empiric methodologies and others based on imaging techniques.

During the last years, numerous 3D reconstruction projects have been crowned with success. Some of them have produced impressive results by introducing the fusion of different digitization methodologies [1]-[8]. The main purpose behind such a combination is no other than acquiring the best of the different approaches. In other cases this fusion aims at the automation of the digitization phase. Nonetheless, it still remains a highly active research area, as there is still no panacea methodology to comply with the multiformity of cultural heritage.

In this paper we present methods that are available today for 3D digitization of architectural heritage and we present two case studies of real-world digitization projects involving two different categories of architecture, monuments and urban areas. The first case study deals with the 3D digitization and representation of two Byzantine churches of Mani in Peloponnesus, Greece. It is a joint attempt of three different Institutions in Greece, the Cultural and Educational Technology Institute, the 5th Ephorate of

$1 \quad$ Text ratified by the 11th ICOMOS General Assembly, held in Sofia, Bulgaria, 5-9 October 1996. 
Byzantine Antiquities and the Department of Architectural Engineering of Democritus University of Thrace. The second case regards the digitization and 3D representation of a part of the old town of Kavala in North-Eastern Greece. It is a collaboration of the Cultural and Educational Technology Institute and the Department of Architectural Engineering of Democritus University of Thrace.

\section{THREE-DIMENSIONAL (3D) DIGITIZATION}

Complete recording of Cultural Heritage is a multidimensional process. It does not only address the problem of three-dimensional (3D) digitization of monuments but involves all the aspects of this new digital content management, representation and reproduction, thus affecting the whole life cycle of the digital cultural content. Due to the complexity of the digitization needs that emerge from the monuments themselves, it is not possible to identify a single methodology which is applicable to all cases. Five main processes can be identified in digital recording. These processes are shown graphically in Figure 1. Every process requires the usage of advanced algorithms, new hardware and more sophisticated software implementations.

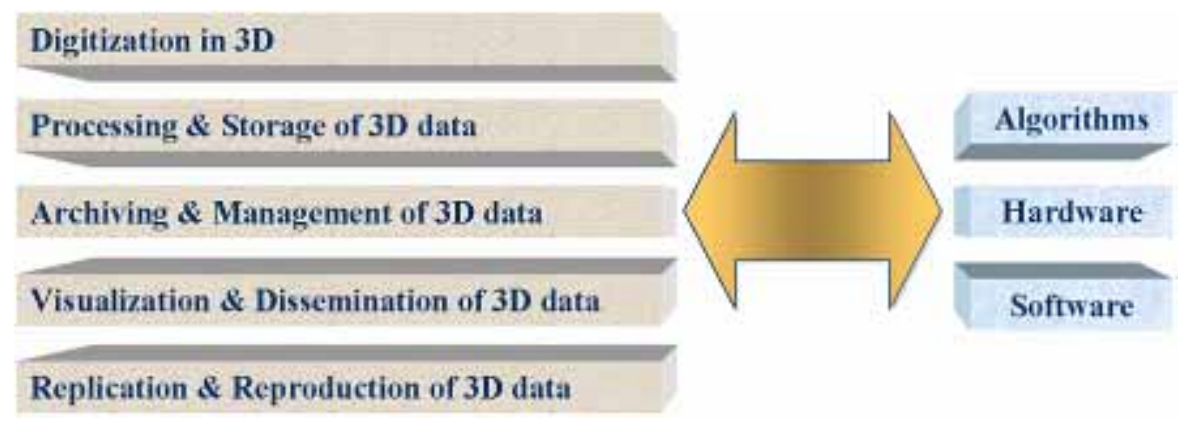

Figure 1. Complete recording of cultural heritage

Nowadays, 3D digitization is considered as the first step of a complicated process that leads to the complete recording of a monument. This process consists of multiple steps and exhibits variations in accordance with specific application requirements. Due to the complexity of the digitization needs that emerge from the actual objects, there is a plethora of methods and technologies. The scope of every such technique is to address successfully a particular type or class of monuments, or to fulfill particular demands and needs of a 
specific digital recording project (i.e. complete recording for archiving, digitization for presentation, digitization for commercial and promotional exploitation).

\subsection{Digitization of monuments}

$3 \mathrm{D}$ digitization of cultural content can be mainly categorized by the size of objects it is applied to. Due to technical limitations and application requirements, there must be a distinction between the digitization of objects and the digitization of monuments. Digitization of monuments is, in many cases, based on methods that involve traditional surveying techniques (due to scale reasons). In the following paragraphs we briefly review these methods.

\section{Empiric technique}

During the recording of monuments by using the empiric technique, measurements of distances between characteristic points on the surfaces of the monument are taken by hand (Figure 2). The coordinates are defined on an arbitrary coordinate system on a plane surface of the monument. This method is simple, productive, portable and of low cost. On the other hand, it is of low accuracy and rather demanding in terms of time of physical presence on site. It can be successfully applied when a monument has low façade complexity, or there is a need for recording a sectional plan or sections of the interiors [9].
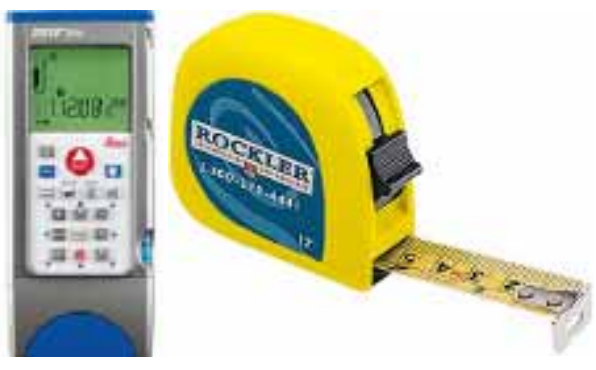

Figure 2. Instruments for empiric techniques of 3D digitization of monuments

\section{Surveying technique}

Surveying techniques implement a 3D orthogonal coordinate system by using complicated and high accuracy measuring devices (Figure 3). This method, mainly, uses a Total Station, a device for measuring angles and distances of characteristic points on the surface of the monument, which are further on transformed into coordinates in reference to the initial orthogonal coordinate system. The main advantage 
of such a method is its high accuracy and objectivity of its measurements.. It is not only reliable but it also provides an easy process of the measuring data. Although the method requires long periods of physical presence on site, it is the only one that can be used under challenging situations such as complex morphology of the monument and difficulty to access specific areas of the site. It is though of as the ideal method for producing high accuracy models of scale 1:50 or smaller [9].

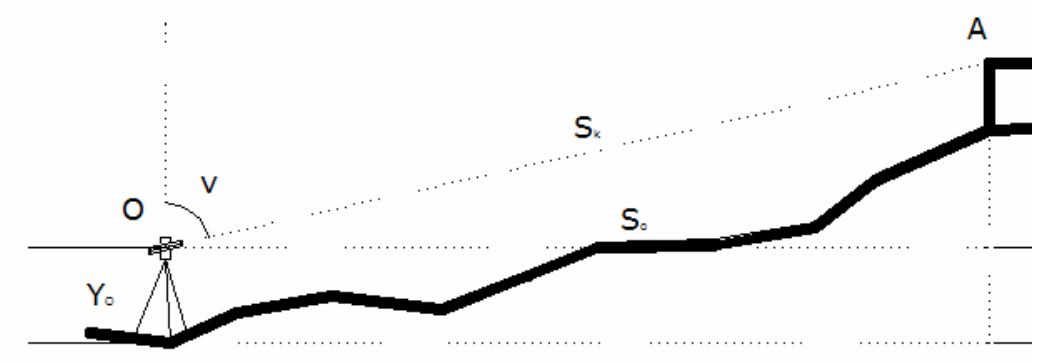

Figure 3. Topographic measurements

\section{LASER SCANNING TECHNIQUES}

Laser scanners can actually be considered as advanced geodesic stations and can be used to measure topographic quantities. These devices can be used to measure the direction of a fictional optical line joining the characteristic points on a surface of a monument to a reference point on the measuring device (Figure 4). Additionally these scanners can estimate their distance from these points. By applying the known triangulation principle they produce Cartesian coordinates automatically.
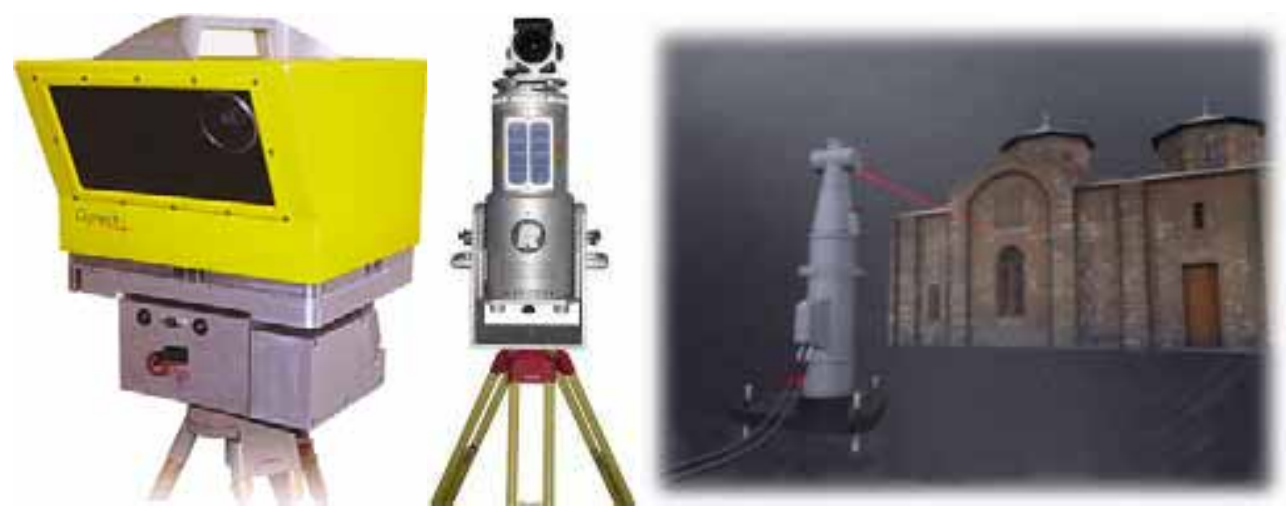

Figure 4. Laser scanning devices and representation of laser scanning process 
The main advantages are high accuracy and productivity, as well as the large volume of produced measurement data in a fraction of time (Figure 5). It is both reliable and objective. But it is also considered as a high cost method with portability and autonomy difficulties. It can be applied on almost any monument digitization, but the measurements' accuracy is affected by very bright light [10].
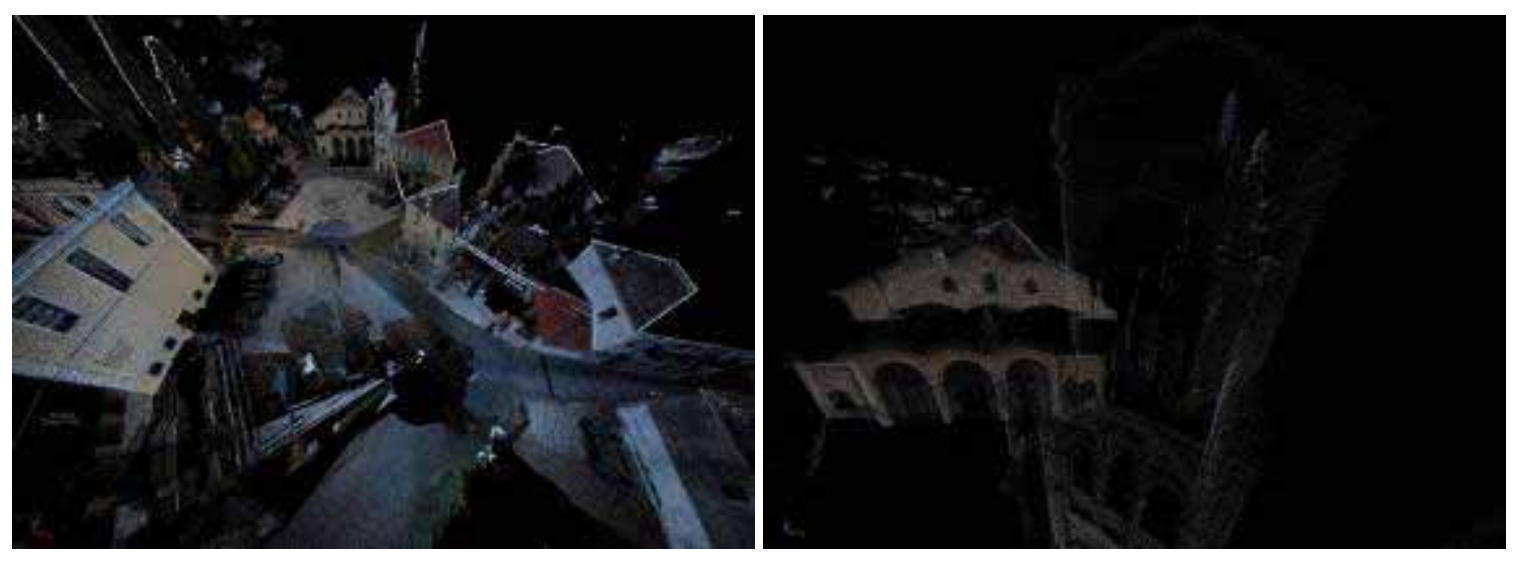

Figure 5. Point clouds (with color information) produced by laser scanning of urban areas (Courtesy Guerra F. and associates, International Conference "Imaging Culture", Xanthi, Greece, March 2006)

\section{PHOTOGRAMMETRY}

Common digital photos can be used, under suitable conditions, for measurements that can be of the accuracy obtained by the topographic methods. By applying orientation processes and transformations of digital photogrammetry it is possible to deduce 2D or 3D coordinates from one or two photos (Figure 6). The method is objective and reliable and can be aided by CAD software. It is relatively simple and of low cost. On the other hand it has to be combined with topographical or empiric measurements and the final outcome is a function of the time spent. It can be used for complex objects with high surface detail, but since it is based on photos, there is a need for adequate space (distance from the monument). It is also useful when direct access or contact to the monument is prohibited. It can be used to record stages of the monument during different time periods. When combined with accurate measurements it can produce models of high accuracy for scales of 1:100 and even higher [9],[11],[12].

Photogrammetric modeling of buildings having great cultural value is becoming a common task during the last years [13]. It is not only a correct way of buildings' documentation but also a fast and simple way to the restitution of their basic geometric and thematic information. 

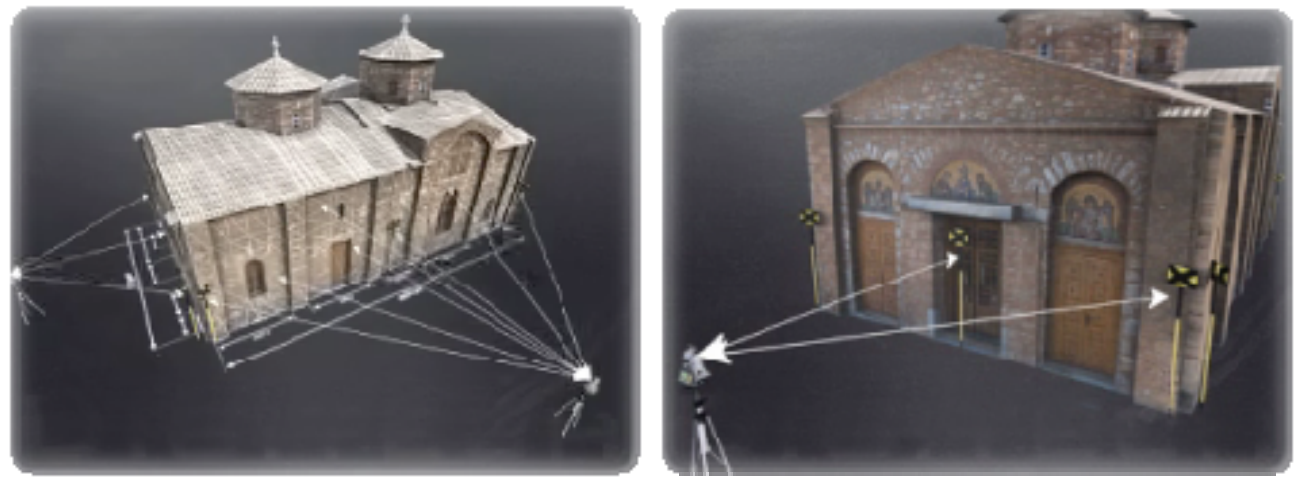

Figure 6. Representation of the photogrammetric method

\section{CASE STUDIES: DIGITIZATION OF MONUMENTS AND URBAN AREAS}

In this part of the paper we present two case studies of real-world digitization projects involving monuments and urban areas. The first, case study is a joint attempt for the 3D digitization and representation of two Byzantine churches in one of the richest in Byzantine antiquities sites in Greece, Mani (Peloponnesus). The second case study is an attempt of the Cultural and Educational Technology Institute to digitize and represent in 3D a part of the old city of Kavala in North Greece, within the framework of a regional digitization project.

\subsection{MONUMENTS: THE CASE OF BYZANTINE CHURCHES IN MANI, PELOPONNESUS [3]}

In this case study we present the experience gained and the results of our project of digitizing two Byzantine churches of Mani Episkopi at Stavri and Agios Nikolaos at Kelefa of Mani, Peloponnesus, Greece (Figure 7). Although laser scanning [10],[14] would be the most appropriate method for the generation of the textured models of the two monuments, the photogrammetric modeling was chosen instead, due to low budget and the adequacy for medium accuracy.

The 3D model of the outer surfaces of both churches has been acquired by using a set of photographs with two commercial photogrammetric software packages, Photomodeler and 3D Builder. The photo camera that was used for capturing the images was a 6.3 Mpixel NIKON D1X dSLR camera equipped with a $17 \mathrm{~mm}$ wide-angle lens. The camera was properly calibrated using Photomodeler. Horizontal and vertical 
measurements of distances between well-distinguished and properly distributed feature points on the 3D objects gave the appropriate control for the registration of the images in the 3D space and the final calculation of the 3D model. The overall accuracy of the derived 3D model was $0.5-0.8 \%$. The generated models were accurate enough for the production of satisfactory solid models that would be the basis of the textured virtual reality models of the two monuments (Figure 8). The surface models were imported into 3D Studio Max for texture mapping. The final texture images of the planar outer surfaces were produced using Microstation/IRASC. Photogrammetric image processing has also been applied for the creation of the photomosaics of the inner textures.

The photogrammetric processing was carried out rather easily and with no particular problems using a small number of measurements and a bundle of images covering all around the entire objects (Figure 9). Additional measurements gave the appropriate validation points during the photogrammetric processing.
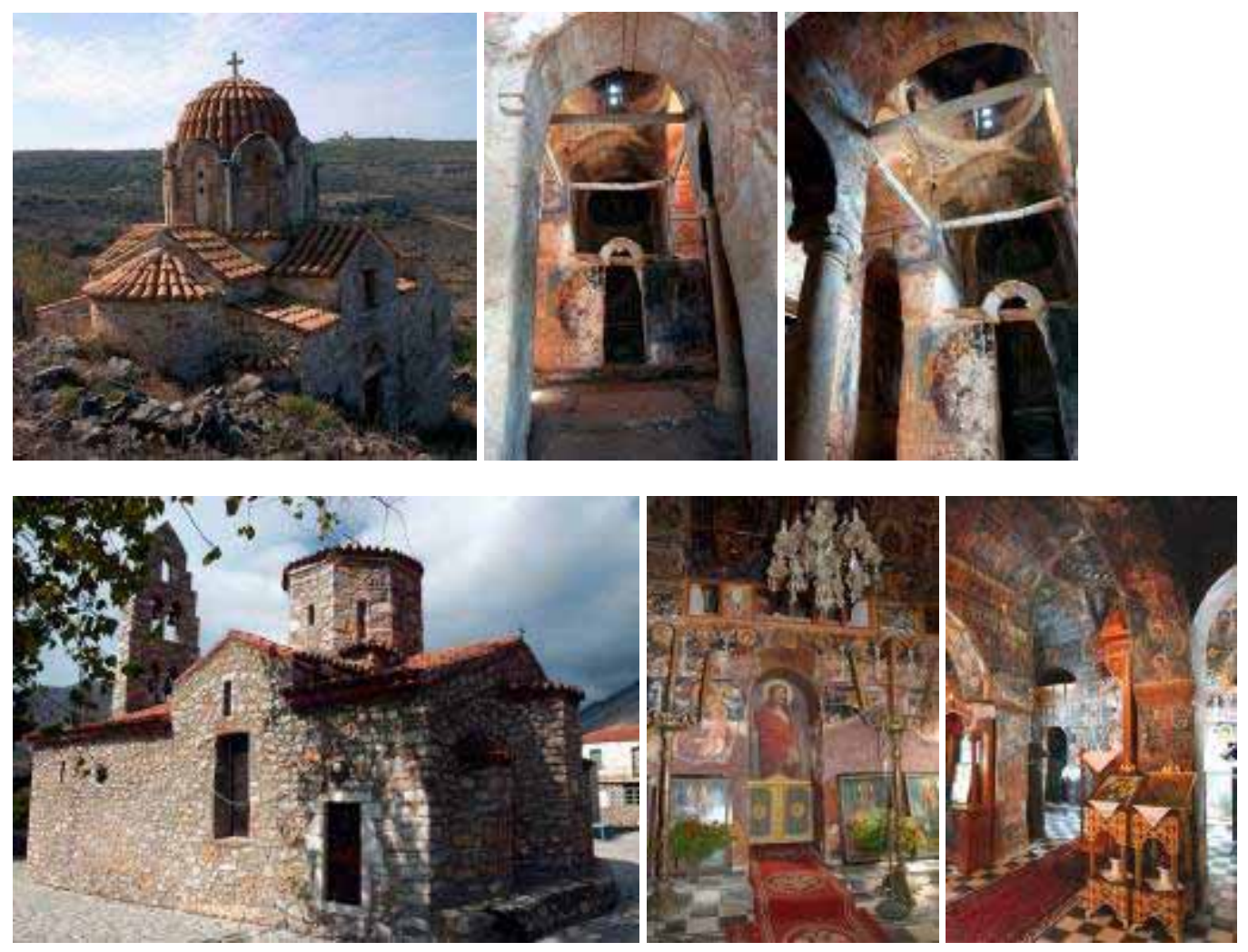

Figure 7. The temples are characterized of beautiful decorative constructions and paintings of great archaeological and relational importance. Episkopi above and Agios Nikolaos bellow. 


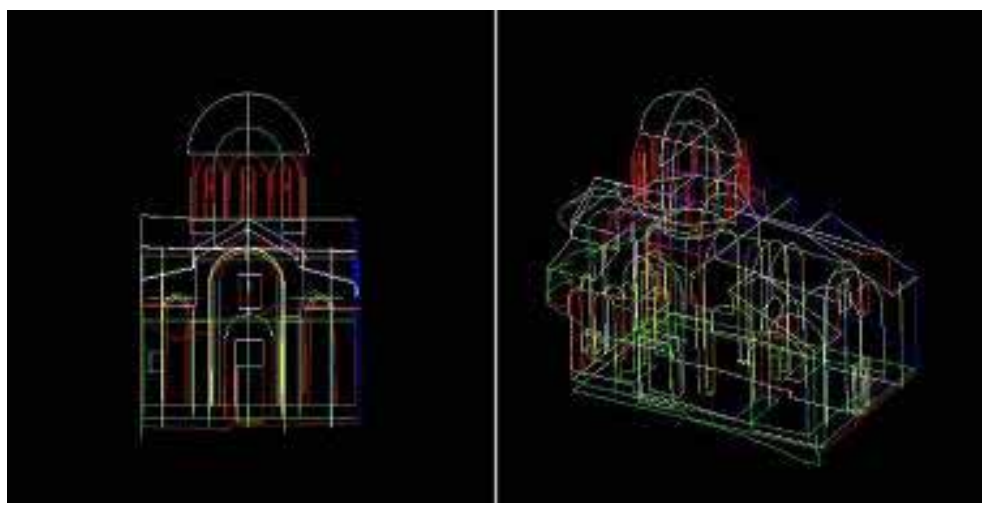

Figure 8. The wireframe model (front and isometric view) of one of the churches
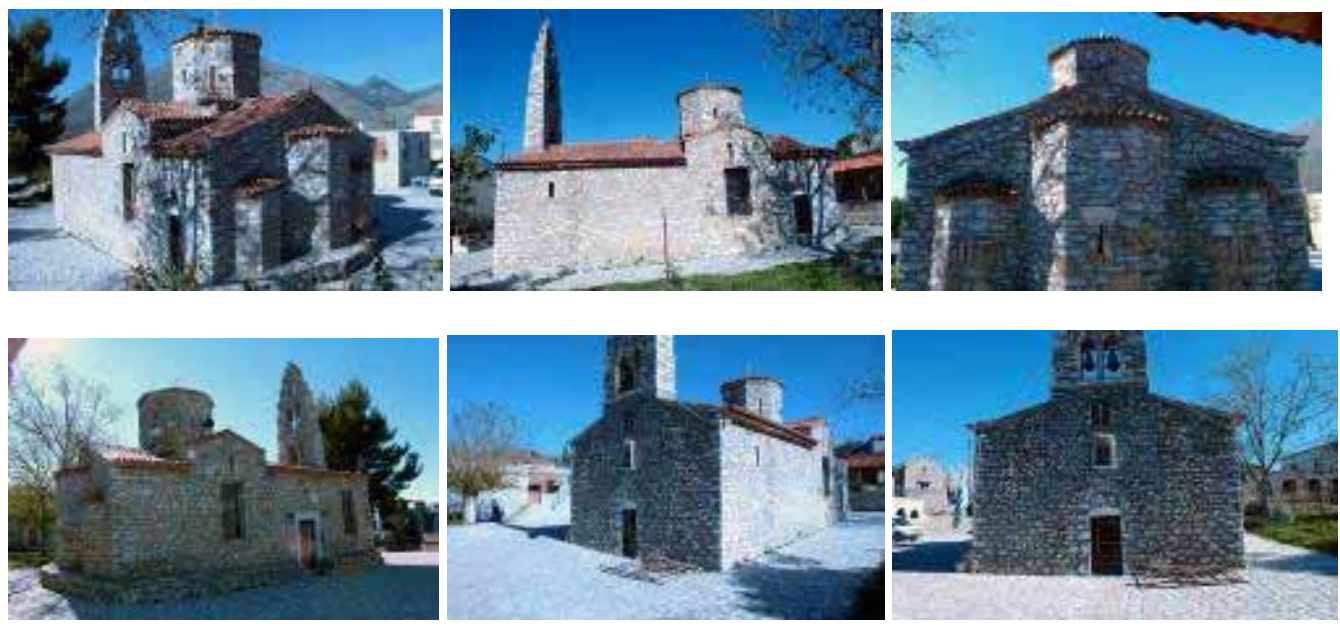

Figure 9. The photogrammetric processing was carried out rather easily using a bundle of images covering all around the entire buildings - here shown for Agios Nikolaos

In Figure 10, 3D reconstructions of the exteriors of the two monuments are shown with reconstructions of the surroundings in order to provide a realistic representation of both the monuments and the topology of the area. Accordingly, Figure 11 depicts 3D reconstructions of the interiors of the two churches.

Three versions of the reconstruction have been produced, in order to provide bandwidth compatibility for the efficient transmission of the 3D data over the Internet. These versions include a high, a medium and a low quality texture representation in order to significantly reduce the required transmission time. Table 1 lists the sizes of texture and geometry for the three versions. Textures represent the greatest percentage of the data that have to be transmitted, and as of this, the textures are reduced both in terms of compression 
and resolution. Geometry, which is in the form of VRML (textual form), it is compressed using the typical gzip compression, that is supported by the standard.
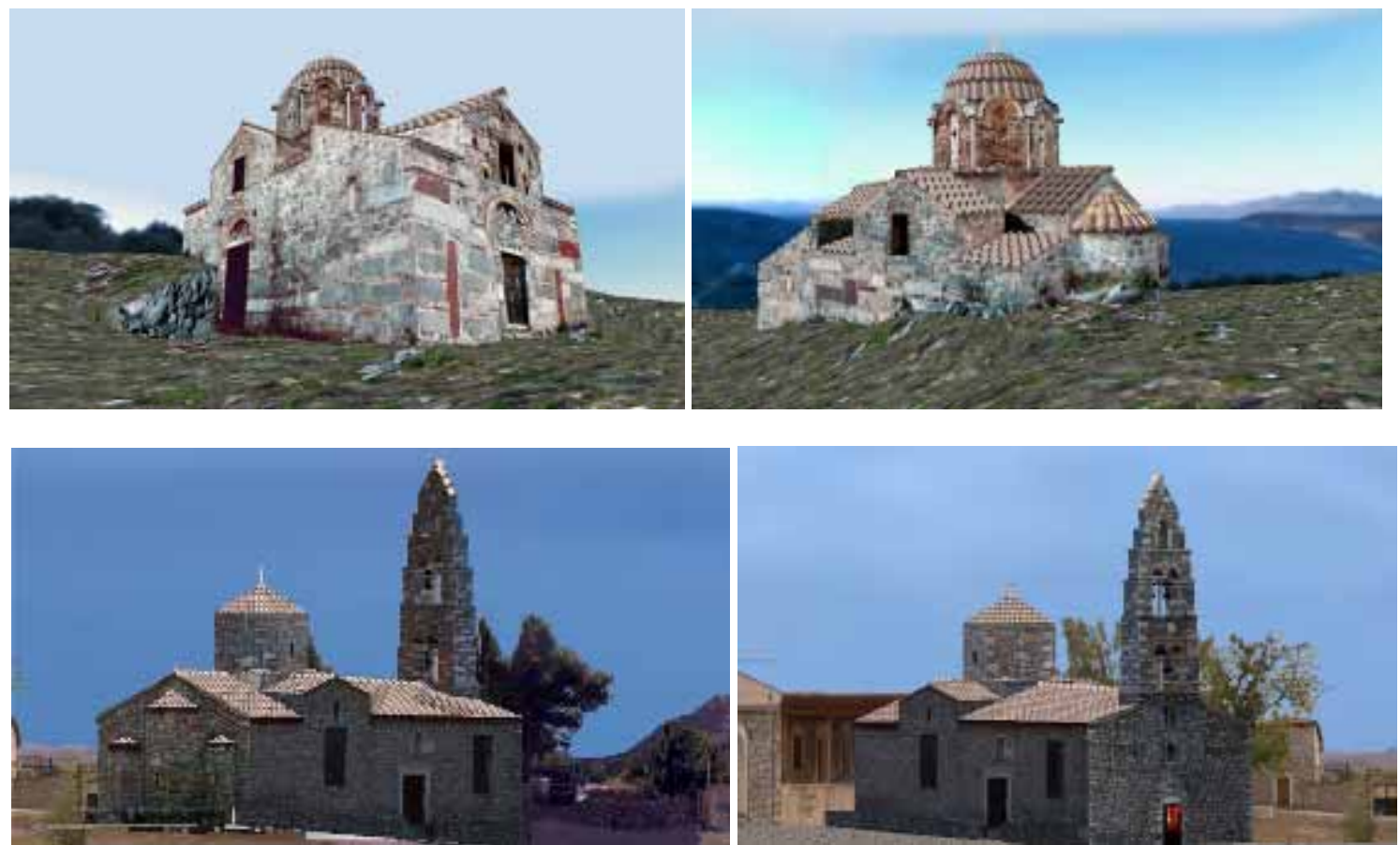

Figure 10. 3D reconstructions of the exteriors of the two churches with surroundings - Episkopi (top), Agios Nikolaos
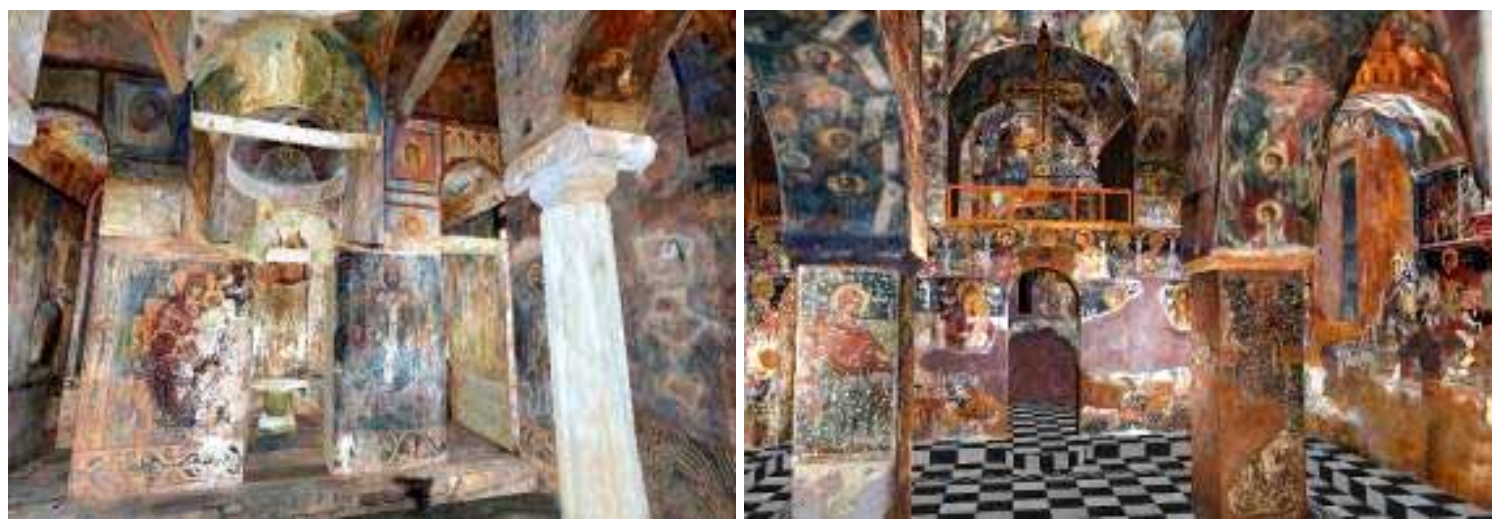

Figure 11.3D reconstructions of the interiors of the two churches - Episkopi (left), Agios Nikolaos 
Data reductions lead to reductions in quality, which is typically measured by metrics such as the PSNR

[15]. For this case study, the strategy for measuring the PSNR is as follows: screenshots are taken from the same viewpoints of each of the three versions and PSNR measurements between the medium and low against the high quality version are collected. The overall average PSNR of the medium compared to the high quality was $36.64943 \mathrm{~dB}$ for Episkopi and $37.03971 \mathrm{~dB}$ for Agios Nikolaos, while the overall average PSNR of the low compared to high quality was $34.00286 \mathrm{~dB}$ for Episkopi and $31.18902 \mathrm{~dB}$ for Agios Nikolaos. Typical download times are listed in Table 2. It should be noted that the medium and high quality of both the 3D models were produced mainly for offline usage, due to the need for detailed presentation of the inner structures and wall paintings.

Table 1: Size of texture and geometry for the three versions of the 3D reconstructions.

\begin{tabular}{|l|l|l|l|}
\hline & High (bytes) & Medium (bytes) & Low (bytes) \\
\hline \multicolumn{4}{|c|}{ Episkopi } \\
\hline Textures & 85732105 & 9431862 & 4497559 \\
\hline Geometry (VRML-gzipped) & 746440 & 645357 \\
\hline \multicolumn{5}{|c|}{ Agios Nikolaos } \\
\hline Textures & 117374211 & 29239068 & 6453 \\
\hline Geometry (VRML-gzipped) & 276833 &
\end{tabular}

Table 2: Typical ideal download times over different networks.

\begin{tabular}{|l|l|l|l|l|l|l|}
\hline & $\begin{array}{l}\text { 56Kbps } \\
\text { PSTN }\end{array}$ & $\begin{array}{l}\text { 64Kbps } \\
\text { ISDN }\end{array}$ & $\begin{array}{l}\text { 128kbps } \\
\text { ISDN }\end{array}$ & $\begin{array}{l}\text { 512Kbps } \\
\text { DSL }\end{array}$ & $\begin{array}{l}\text { 1Mbps } \\
\text { DSL }\end{array}$ & $\begin{array}{l}\text { 2Mbps } \\
\text { DSL }\end{array}$ \\
\hline Low & $00: 11: 22$ & $00: 09: 56$ & $00: 04: 58$ & $00: 01: 14$ & $00: 00: 37$ & $00: 00: 18$ \\
\hline Medium & $00: 23: 06$ & $00: 20: 13$ & $00: 10: 06$ & $00: 02: 31$ & $00: 01: 15$ & $00: 00: 37$ \\
\hline
\end{tabular}




\begin{tabular}{|l|l|l|l|l|l|l|}
\hline & $\begin{array}{l}\text { 56Kbps } \\
\text { PSTN }\end{array}$ & $\begin{array}{l}\text { 64Kbps } \\
\text { ISDN }\end{array}$ & $\begin{array}{l}\text { 128kbps } \\
\text { ISDN }\end{array}$ & $\begin{array}{l}\text { 512Kbps } \\
\text { DSL }\end{array}$ & $\begin{array}{l}\text { 1Mbps } \\
\text { DSL }\end{array}$ & 2Mbps \\
DSL \\
\hline High & $03: 24: 46$ & $02: 59: 11$ & $01: 29: 35$ & $00: 22: 23$ & $00: 11: 11$ & $00: 05: 35$ \\
\hline \multicolumn{7}{|c|}{ Agios Nikolaos } \\
\hline Low & $00: 16: 01$ & $00: 14: 01$ & $00: 07: 00$ & $00: 01: 45$ & $00: 00: 52$ & $00: 00: 26$ \\
\hline Medium & $01: 10: 16$ & $01: 01: 29$ & $00: 30: 44$ & $00: 07: 41$ & $00: 03: 50$ & $00: 01: 55$ \\
\hline High & $04: 40: 07$ & $04: 05: 06$ & $02: 02: 33$ & $00: 30: 38$ & $00: 15: 19$ & $00: 07: 39$ \\
\hline
\end{tabular}

\subsection{URBAN AREA: THE CASE OF A PART OF THE OLD CITY OF KAVALA}

In this case study we demonstrate the usage of a low-cost open-source based 3D digitization pipeline of a small part of the old town of the city of Kavala (Eastern Macedonia, Greece). It is a picturesque area with cobbled roads that lead to an appealing example of Turkish house architecture of the 18th century (where Mehmet Ali Paşa, founder of the last Egyptian royal line was born) and a Christian Orthodox church dedicated to Virgin Mary. Both monuments are located within the walls of a Byzantine castle.

The proposed pipeline includes the usage of the following tools:

- Panorama Tools: a collection of free software programs for creating panoramas from multiple images. The core software was originally written by Professor Helmut Dersch of the Fachhochschule Furtwangen.

- Hugin: a cross-platform open source panorama photo stitching program developed by Pablo d'Angelo and others. It is, actually, a GUI front-end for Panorama Tools.

- The GNU Image Manipulation Program or just the GIMP: a raster graphics editor for creating and processing raster graphics with support for vector graphics. The project was initiated in 1995 by Spencer Kimball and Peter Mattis and is now maintained by a group of volunteers, licensed under the GNU General Public License.

- Blender: an open source software for 3D modeling, animation, rendering, post-production, interactive creation and playback. Available for all major operating systems under the GNU General Public License. 
- Voodoo Camera Tracker: a camera tracking tool for the integration of virtual and real scenes. This non-commercial software tool is developed for research purposes at the Laboratorium fuer Informationstechnologie, University of Hannover.

The reconstructions that can be produced within the framework defined by these tools are to be considered mainly for promotional purposes and not for digital archival or scientific study. Figure 12 depicts the phases of the pipeline. The data acquisition phase (defined by the dashed line) denotes the actual fieldwork. At this phase a thorough study of the target field is required in order to decide on which of the geometric features of the buildings and surroundings are going to be modeled as well as to decide on the measurements and photo shooting.

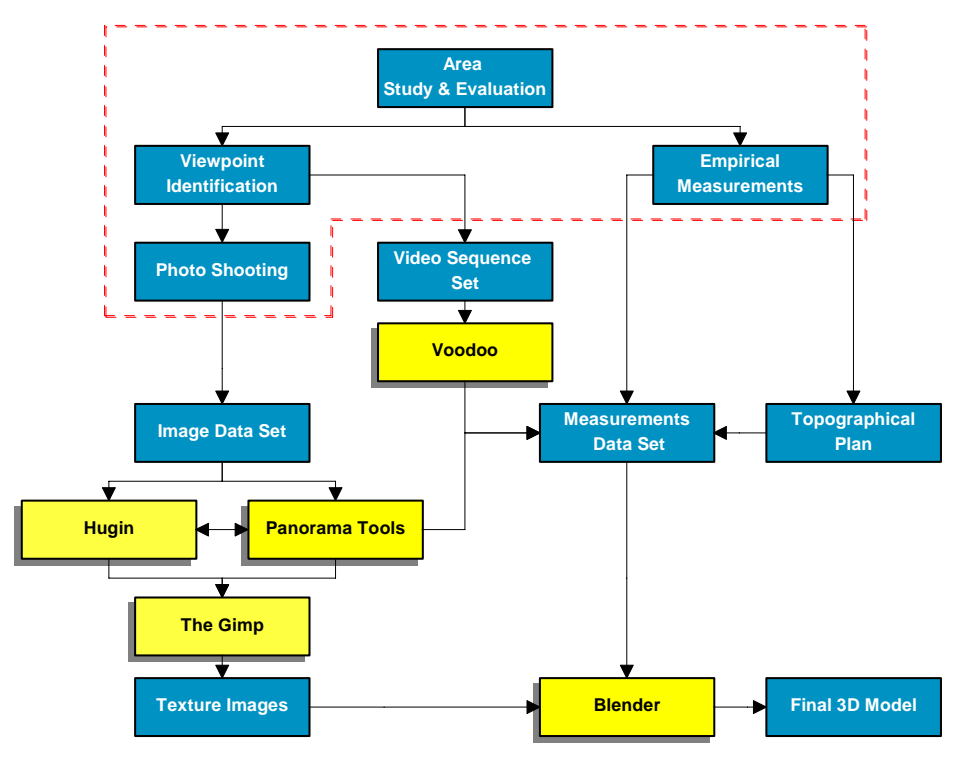

Figure 12. The pipeline for 3D digitization and reconstruction of urban areas based on open source tools

The photographic dataset, formed during the photo shooting, should cover as much as possible visible information. The purpose of this dataset (including video) is twofold. Hugin can be used for image rectifications [16] (Figure 13). 


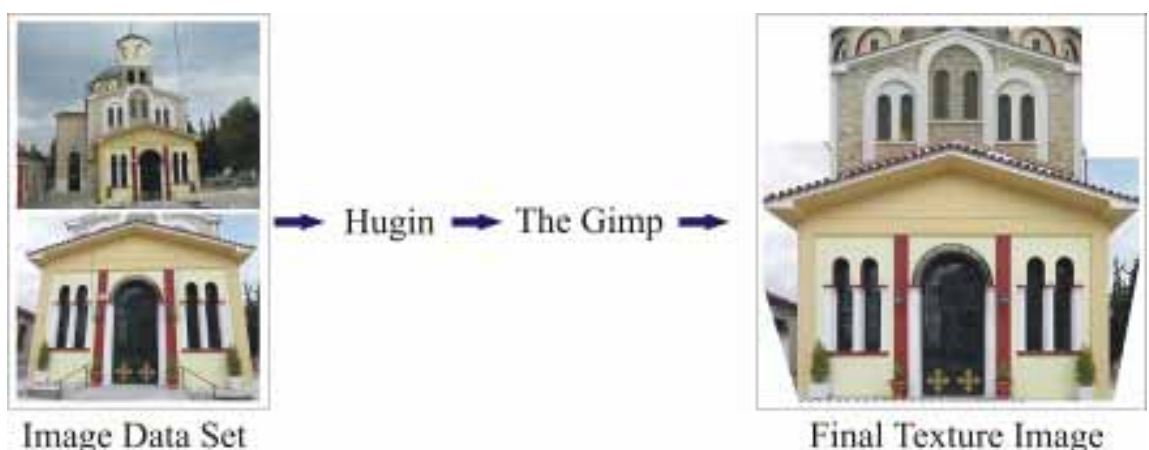

Figure 13. Image rectification

Panaroma Tools can provide a low density triangulated mesh based on the shape-from-stereo technique [17],[18] - which is usually used for deducing the 3D geometry of objects by exploiting two cameras or photos from different angles - by using the ptpicker and ptstereo software components. The output mesh doesn't reflect real dimensions but only relative distances. The measurements acquired according to the empiric method aid in transforming those relative quantities to real-world dimensions. Voodoo Camera Tracker can provide a denser 3D point cloud based on feature points automatically detected and extracted from a video sequence. Both 3D data sets provide valuable information as they can be imported into Blender.

A 3D approximation of the area can be modeled by combining data from the empiric method, a topographical plan and the point clouds. Finally, the rectified images are used for texture mapping. Alternatively, Panaroma tools and Hugin can be used to create image panoramas that can be mapped on planar surfaces which act as backgrounds or as areas where modeling is not possible. The versatility of the pipeline allows different approaches to be followed during the modeling phase.

A total time of 500 person-hours over a two month period was allocated for the project. Since, the development of the 3D model was focused on producing a web based virtual walkthrough of the cultural district, greater efforts were put to the construction of medium geometry models with high texture details. As known, texture maps can provide the illusion of details in low geometry models [19]. Experiments have shown that pictorial realism plays an important role in an immersive VR system [20].

The main problems faced during the photo shooting were:

- Viewpoint limitations and occlusion 
- Lenses distortions due to the need for wide angle lenses

- Weather conditions (heavy shadow casting and high contrast)

- $\quad$ Long times of physical presence (field work)

- Unexpected events, like distractions from locals

A total of 900 photos were taken using an 8 Mpixel digital camera along with 192 measurements based on empiric method. Additional topographical and historical information were also collected and related with the architectural elements of the area. The final model of the reconstructed area (Figure 14) is includes a total of 11673 vertices that define a set of 17832 facets. Such geometric complexity can easily be handled even by low-end graphics cards.
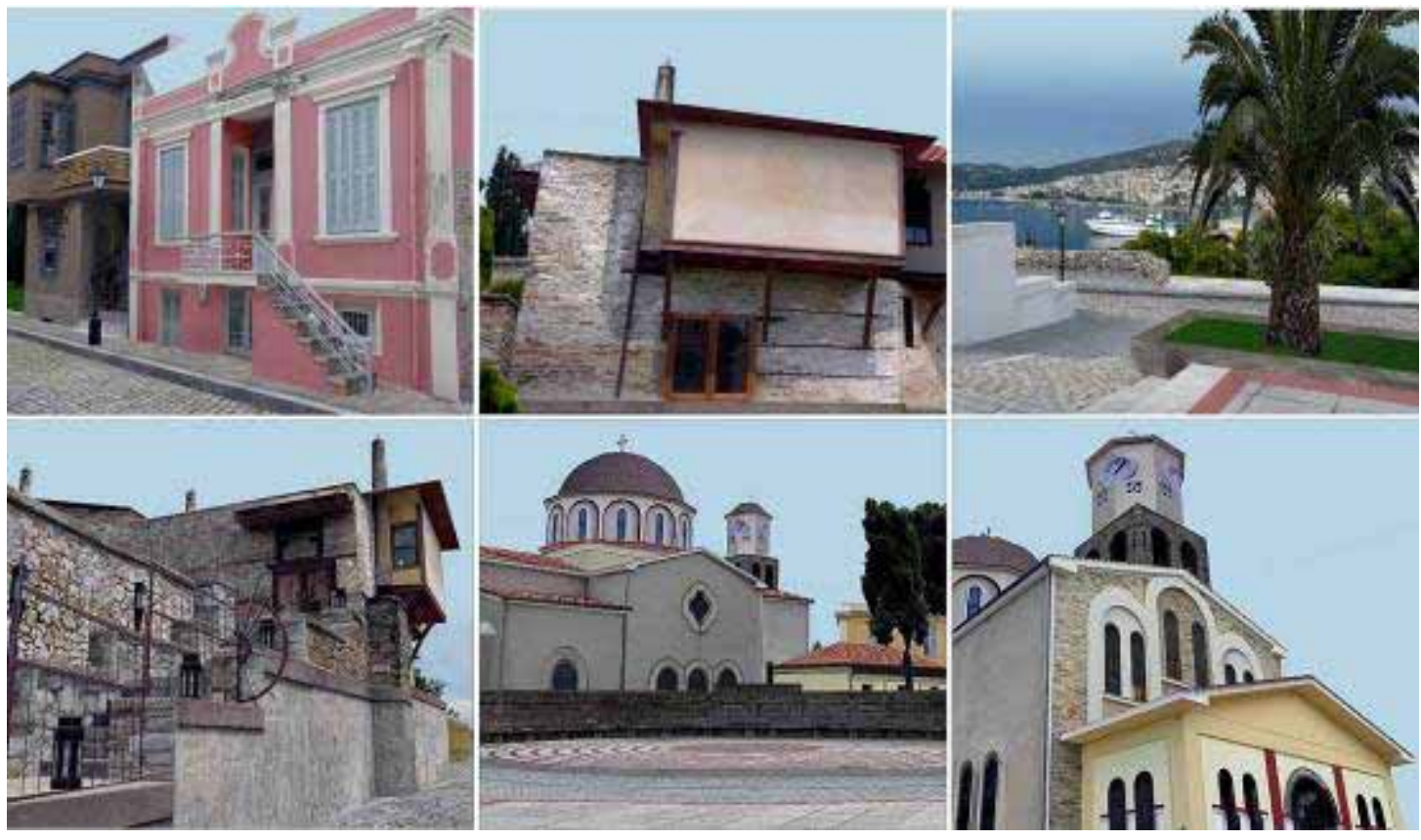

Figure 14. Screenshots during a virtual tour inside reconstructed area

Once again, in order to provide bandwidth compatibility for the efficient transmission of the 3D data over the Internet, three versions of the reconstruction have been produced. These versions include a high, a medium and a low quality texture representation in order to significantly reduce the required transmission time. In Table 3, the sizes of texture and geometry are listed for each of the three versions. As shown, textures represent the greatest percentage of the data that have to be transmitted, and as of this, the textures 
are the target of data reduction both in terms of compression and resolution reduction. Geometry, which is in the form of VRML (textual form), it is compressed using the typical gzip compression, that is supported by the standard.

Table 3: Size of texture and geometry for the three versions of the 3D reconstructions.

\begin{tabular}{|l|l|l|l|}
\hline & High (bytes) & Medium (bytes) & Low (bytes) \\
\hline Textures & 28630685 & 5519871 & 2266882 \\
\hline Geometry (VRML-gzipped) & \multicolumn{3}{|c|}{208089} \\
\hline
\end{tabular}

These data reductions, as expected, lead to reductions in quality, which is typically measured by metrics such as the PSNR. For this case study, the strategy for measuring the PSNR is as follows: screenshots are taken from the same viewpoints of each of the three versions and PSNR measurements between the medium and low to the high quality version are collected. The overall average PSNR of the medium compared to the high quality was $38.89640 \mathrm{~dB}$, while the overall average PSNR of the low compared to high quality was $31.41654 \mathrm{~dB}$. Typical download times are listed in Table 4.

Table 4: Typical ideal download times over different networks.

\begin{tabular}{|l|l|l|l|l|l|l|}
\hline & $\begin{array}{l}\text { 56Kbps } \\
\text { PSTN }\end{array}$ & $\begin{array}{l}\text { 64Kbps } \\
\text { ISDN }\end{array}$ & $\begin{array}{l}\text { 128kbps } \\
\text { ISDN }\end{array}$ & $\begin{array}{l}\text { 512Kbps } \\
\text { DSL }\end{array}$ & 1Mbps DSL & 2Mbps DSL \\
\hline Low & $00: 05: 53$ & $00: 05: 09$ & $00: 02: 34$ & $00: 00: 38$ & $00: 00: 19$ & $00: 00: 09$ \\
\hline Medium & $00: 13: 38$ & $00: 11: 55$ & $00: 05: 57$ & $00: 01: 29$ & $00: 00: 44$ & $00: 00: 22$ \\
\hline High & $01: 08: 39$ & $01: 00: 04$ & $00: 30: 02$ & $00: 07: 30$ & $00: 03: 45$ & $00: 01: 52$ \\
\hline
\end{tabular}

\section{CONCLUSION}

3D digital recording of Cultural Architecture is a multi-dimensional process, which depends on various factors and as well as the main purpose of recording. Main aims of 3D recording are in the preservation and 
promotion of the cultural content. In this work we made an attempt to summarize most of the methods available today for 3D digitization that can be applied to cultural architecture recording. Two case studies were provided in order to share the knowledge and experience gained by two 3D digitization projects, one for monuments and one for urban areas.

\section{References}

1. Sormann M., Fast and Detailed 3D Reconstruction of Cultural Heritage, Proc. International Workshop on Vision Techniques applied to the Rehabilitation of City Centers, Oct. 25-27, 2004, Lisbon, Portugal.

2. Kersten, 3D Acquisition, Modeling and Visualization of North German Castles by Digital Architectural Photogrammetry, Proc. Scene Modeling and Virtual Reality, XXth ISPRS Congress, Jul. 12-23, 2004, Istanbul, Turkey.

3. Tsioukas V., Tsirliganis N., Pavlidis G., Arnaoutoglou F., Chamzas Ch., Mpakourou E. and Mexia A., Photogrammetric Modeling of Byzantine Churches, CIPA 2005, XX International Symposium, Sep. 26 - Oct. 01, 2005, Torino, Italy.

4. El-Hakim F. Sabry, Detailed 3D Reconstruction of Large-Scale Heritage Sites with Integrated Techniques, IEEE Computer Graphics and Applications, 2004, Vol. 24, No. 3, pp. 21-29.

5. Van Gool L., The MURALE Project: Image-Based 3D Modeling for Archaeology. Virtual Archaeology, Proc. of the VAST2000 Euroconference, Arezzo, Italy, Nov. 2000, pp.53-63.

6. Beraldin J.-A., Combining 3D Technologies for Cultural Heritage Interpretation and Entertainment, SPIE: Electronic Imaging 2005, Videometrics IX. San Jose, Jan. 16-20, 2005, California, USA.

7. Sgrenzaroli M., Cultural Heritage 3D Reconstruction using High Resolution Laser Scanner: New Frontiers Data Processing, CIPA 2005 XX International Symposium, Sep. 26 - Oct. 01, 2005, Torino, Italy. 
8. Tsirliganis N., Pavlidis G., Koutsoudis A., Papadopoulou D., Tsompanopoulos A., Stavroglou K., Politou E. and Chamzas C., Integrated Documentation of Cultural Heritage through 3D imaging and multimedia database, Proc. of VAST2001: Virtual Reality, Archaeology, and Cultural Heritage, Nov. 28-30, 2001, Glyfada, Athens, Greece.

9. Livieratos E., Empiric, Topographic or Photogrammetric recording? Answers to properly phrased questions, Proceedings of the congress, "Terrestrial Photogrammetry and Geographic Information Systems for the documentation of the National Cultural Heritage", 1992, Thessaloniki, Greece.

10. Boehler W. and Marbs A., 3D Scanning Instruments, Proceedings Of The CIPA WG 6 International Workshop, “On Scanning For Cultural Heritage Recording”, 2002, Corfu, Greece.

11. Hanke K. and Grusenmeyer P., Architectural Photogrammetry: Basic theory, Procedures, Tools, ISPRS Commission 5 Tutorial, Sep. 2002, Corfu, Greece.

12. Tsioukas, V. and Patias, P., Low Cost 3D Visualization and Measuring 'Tool' in the Service of Archaeological Excavations, Computer Applications and Quantitative Methods in Archaeology Conference, "The Digital Heritage of Archaeology", 2002, Heraklion, Greece.

13. Balodimos D., Lavvas G., and Georgopoulos A., Wholly Documentation Holly Monuments, Proceedings of the CIPA 2003 International Symposium: "New Perspectives to Save Cultural Heritage”, 2003, Turkey, pp. 502-506.

14. Beraldin J.-A., Picard M., El-Hakim S.F., Godin G., Latouche C., Valzano V. and Bandiera A., Exploring A Byzantine Crypt Through A High-Resolution Texture Mapped 3D Model: Combining Range Data And Photogrammetry, Proceedings of the CIPA WG6 International Workshop "Scanning of Cultural Heritage", 2002, Greece, pp. 65-70.

15. Kang L. W. and Leou J. J., A new error resilient coding scheme for JPEG image transmission based on data embedding and vector quantization, in Proc. of 2003 IEEE Int. Symposium on Circuits and Systems (ISCAS2003), May 2003, Bangkok, Thailand, vol. 2, pp. 532-535. 
16. Suveg I., 3D reconstruction of building models, International Archives of Photogrammetry and Remote sensing, 2000, Vol.33, Part B2, part 2, pp 538-545.

17. Scharstein D. and Szeliski R., A Taxonomy and Evaluation of Dense Two-Frame Stereo Correspondence Algorithms, International Journal of Computer Vision, Apr. 2002, Vol. 47, No. 13.

18. Bertozzi M., Broggi A., Conte G. and Fascioli A., Stereo-Vision System performance analysis, Enabling Technologies for the PRASSI Autonomous Robot, ISBN 8882860248, Jan. 2002, ENEA, Rome, Italy, pp. 68-73.

19. Rushmeier H. E. and Rogowitz B. E., Perceptual issues in substituting texture for geometry, $\underline{\text { In }}$ Proc. of SPIE - Human Vision and Electronic Imaging V, Jun. 2000, Vol. 3959, pp. 372-383.

20. Dongsik C., Jihye P., Kim, G.J., Sangwoo H., Sungho H. and Seungyong L., The Dichotomy of Presence Elements: The Where and What, Proc. IEEE Virtual Reality 2003, Mar. 22-26, 2003, pp. 273- 274. 